\title{
Avaliação do nível de conhecimento de portadores de diabetes mellitus sobre adoçantes
}

\section{Assessment of the level of knowledge of diabetes mellitus patients about sweeteners}

\author{
Tiemi Saito \\ Rackelly Borges Pereiral \\ Mírian Patrícia Castro Pereira Paixão² \\ 1 Discente do Curso de Graduação em Nutrição. \\ Faculdade Católica Salesina do Espírito Santo. \\ Vitória, ES, Brasil. \\ ${ }^{2}$ Doutora em Ciência e Tecnologia de Alimentos. \\ Docente do Curso de Graduação em Nutrição. \\ Faculdade Católica Salesiana do Espírito Santo. \\ Vitória, ES, Brasil. \\ Correspondência / Correspondence \\ Tiemi Saito \\ E-mail: tiemisaitol@gmail.com ou ticasaito@ \\ gmail.com
}

\section{Resumo}

A terapia nutricional é essencial para o controle metabólico em diabéticos. O uso de adoçantes em substituição ao açúcar é comum, pois estes atribuem doçura aos alimentos sem aumentar a glicemia. O objetivo deste trabalho foi avaliar o nível de conhecimento de indivíduos portadores de diabetes mellitus atendidos no Centro de Referência Ambulatorial de Carapina, Serra/ES, quanto ao consumo de adoçantes. Os dados de 100 voluntários diabéticos foram coletados entre agosto e outubro de 2010, através de questionário sobre o consumo desse produto. Verificou-se que o uso de adoçante é frequente na população estudada, principalmente do tipo líquido, em comparação com outros produtos dietéticos, e também foi possível identificar que 91,2\% dos entrevistados passaram a utilizar adoçante somente após o diagnóstico da doença. No que concerne à escolha do adoçante, observou-se que é principalmente influenciada pelo sabor $(52,75 \%)$ e que os mesmos controlam a quantidade utilizada. Dentre os outros produtos dietéticos citados, o refrigerante foi o mais consumido. Também se constatou que 59\% não sabem diferenciar diet de light, e que $63 \%$ não têm o hábito de ler o rótulo dos alimentos. Conclui-se que é necessário ofertar maiores esclarecimentos aos diabéticos quanto ao uso adequado dos adoçantes e produtos dietéticos por parte dos profissionais de saúde, com inclusão de informações nutricionais mais completas e compreensíveis nos rótulos desses produtos.

Palavras-chave: Diabetes Mellitus. Edulcorantes, Educação em Saúde. 


\section{Abstract}

Nutritional therapy is essential for metabolic control among diabetic people. The use of sweeteners instead of sugar is common, as they bring sweetness to foods without increasing glycemia. This study aimed to evaluate the level of knowledge of diabetes mellitus individuals attending the Outpatient Center of Reference the Carapina, Serra/ES, regarding the consumption of sweeteners. Data were collected between August and October 2010 in a sample of 100 diabetic volunteers through a questionnaire on the consumption of this product. The use of sweeteners is frequent in the population, specially the liquid product, compared to other diet products and it was also possible to identify that $91,2 \%$ of the interviewed reported that they only began using sweeteners after the diagnostic. Regarding the choice of a sweetener, it was observed that is mainly influenced by the taste $(52.75 \%)$ and that they control the quantity used. Among other diet products mentioned, the most consumed were soda. Moreover, it was found that $59 \%$ did not know the difference between diet and light, and that $63 \%$ did not have that habit of reading food labels. Therefore, it was concluded that health professionals should provide further clarification to diabetic people about the adequate use of artificial sweeteners and diet products, and include more complete nutritional information and understandable labels on these products.

Key words: Diabetes Mellitus. Health Education. Sweeteners.

\section{Introdução}

O diabetes mellitus (DM) é uma doença metabólica, caracterizada por deficiência total ou parcial da insulina, relacionada ao quadro de hiperglicemia crônica. ${ }^{1,2} \mathrm{O}$ DM pode ser subdividido em diabetes tipo 1, tipo 2, gestacional e outros tipos específicos, segundo a Sociedade Brasileira de Diabetes (2010). ${ }^{3}$

O DM compõe um problema de saúde pública devido a grande frequência na população, mortalidade, complicações, tratamento com alto custo financeiro e social e deterioração significativa da qualidade de vida. ${ }^{4,5}$

É uma das doenças crônico-degenerativas mais prevalentes da população mundial: havia 150 milhões de diabéticos no ano de 2000, com expectativa de serem 300 milhões até o ano $2025{ }^{6}$ 
Já a prevalência no Estado do Espírito Santo, Brasil, foi 11,88\%.7 Tal crescimento se justifica pelo aumento crescente da obesidade, do sedentarismo e da maior longevidade.

É uma doença que requer um tratamento que vise ao controle metabólico, a prevenção de complicações agudas e a redução do risco de complicações no longo prazo, obtendo, assim, um equilíbrio entre medicamentos, dieta e atividade física. ${ }^{8}$ A adesão ao tratamento é considerada um dos aspectos mais desafiadores no cuidado ao paciente diabético. ${ }^{9}$ As novas diretrizes não fazem restrições ao consumo de qualquer tipo de carboidrato, e de acordo com os critérios adotados pela Recommendad Dietary Allowances (RDA), da American Diabetes Association (ADA), a ingestão mínima de carboidratos deve ser de $130 \mathrm{~g} / \mathrm{dia} .{ }^{10}$

Embora o uso de sacarose não piore o controle glicêmico, é importante ressaltar que o consumo de alimentos ricos em açúcar pode ser prejudicial, já que na sua maioria são ricos também em gordura, que aumentam o risco de doenças cardiovasculares e obesidade, além de possuir baixo teor de fibras, vitaminas e minerais. ${ }^{10}$

Pacientes que apresentam DM e restringem o consumo do açúcar branco (sacarose) passam a fazer uso de adoçantes e produtos dietéticos, que atribuem doçura aos alimentos com pouca ou nenhuma caloria. Mesmo estes produtos não sendo necessários para o controle metabólico do DM, eles apresentam papel significativo no convívio social e no aspecto psicológico destes pacientes e proporcionam a palatabilidade e o prazer do sabor doce. ${ }^{11}$

Segundo a Portaria no 29, de 13 de janeiro de 1998, da ANVISA, ${ }^{12}$ adoçantes dietéticos são formulados para dietas com restrição de sacarose, frutose e/ou glicose, para atender às necessidades de pessoas sujeitas à restrição da ingestão desses carboidratos. Já os adoçantes de mesa, de acordo com a Portaria no 38, de 13 de janeiro de 1998, ${ }^{13}$ são produtos formulados com a finalidade de conferir sabor doce aos alimentos e bebidas. As matérias-primas sacarose, frutose e glicose não podem ser utilizadas na formulação desses produtos alimentícios.

Torloni ${ }^{14}$ afirma que os adoçantes são edulcorantes (substâncias diferentes do açúcar, que adoçam em pequenas quantidades) e são responsáveis pela boa aparência, durabilidade e textura final de um produto. Desde que usados dentro das recomendações, não fazem mal à saúde, devendo as pessoas atentarem para as recomendações de não haver consumo em excesso..$^{15}$

Os adoçantes são classificados em duas categorias: calóricos e não-calóricos. ${ }^{11}$ Dentre os adoçantes calóricos, encontram-se frutose, sorbitol, manitol, xilitol, lactose, malto-dextrina e sacarose; e os não-calóricos, ciclamato, sacarina, acesulfame-K, esteviosídeo, sucralose e aspartame (combinação da fenilalanina com ácido aspártico). ${ }^{16}$

Há poucos estudos que relatam o conhecimento e o consumo de adoçantes por indivíduos portadores de DM, e considerando a importância desses produtos no tratamento da doença, a 
característica específica de cada adoçante e o fato de não existirem recomendações específicas para sua indicação com relação à terapia nutricional da doença, este estudo tem como objetivo avaliar o nível de conhecimento de indivíduos portadores de diabetes mellitus atendidos no Centro de Referência Ambulatorial (CRA) de Carapina, município de Serra/ES, quanto ao consumo de adoçantes, a fim de conhecer os hábitos alimentares com relação aos adoçantes e orientar o consumo adequado dos mesmos.

\section{Materiais e métodos}

Trata-se de estudo transversal, exploratório e descritivo, realizado no Centro de Referência Ambulatorial (CRA) de Carapina, que funciona anexo à Unidade Básica de Saúde Central de Carapina, do município de Serra/ES, onde ocorre o Programa de Educação e Controle de Diabetes.

A amostra mínima definida para este estudo foi de $5 \%$ do total de indivíduos com DM e cadastrados no programa, que frequentaram o CRA de Carapina para as consultas de rotina que acontecem no horário matutino, entre os meses de agosto a outubro de 2010. Dessa forma, foram selecionados 100 voluntários. A coleta de dados ocorreu após a aprovação do projeto de pesquisa pelo Comitê de Ética em Pesquisa da Faculdade Católica Salesiana do Espírito Santo, atendendo à Resolução no 196/96 do Conselho Nacional de Saúde (Protocolo no 44/2010, Ofício 146/2010/CEP). O instrumento utilizado para coleta de dados foi um questionário semiestruturado, com questões fechadas e abertas, composto por três partes, sendo a primeira os dados sociodemográficos; a segunda, dados relacionados à doença; e por fim, dados referentes ao conhecimento e consumo de adoçantes e produtos dietéticos, totalizando 39 questões que foram adaptadas dos estudos de Sousa ${ }^{17}$ e Oliveira, ${ }^{16}$ com exclusão de algumas questões, para atingir o objetivo deste estudo.

Os dados qualitativos foram apresentados através de frequência absoluta e relativa, e as variáveis quantitativas foram apresentadas em média e desvio padrão. Os dados foram analisados com auxílio do software SPSS, v.17 (2010).

\section{Resultados}

A média de idade do grupo avaliado foi 54,64 \pm 13,88 anos e o tempo de diagnóstico do DM variou entre um mês e 41 anos, com média de 10,58 \pm 8,55 anos.

Observou-se que a maioria dos entrevistados é do sexo feminino (61\%) e classificado, segundo o estado civil, como casada (66\%). Além disso, percebeu-se que 34\% dos pacientes apresentaram renda familiar de um salário mínimo e $50 \%$ estudaram até o ensino fundamental incompleto (tabela 1). 
Tabela 1. Caracterização dos usuários do CRA de Carapina - ES, 2010, segundo sexo, estado civil, renda familiar, escolaridade e tipo de diabetes $(\mathrm{n}=10)$

Frequência absoluta

(n)

Sexo

Masculino

39

61

Feminino

Estado Civil

Solteiro

Casado

Separado

Viúvo

Renda Familiar

Sem renda

66

9

14

$\leq 1 \mathrm{SM}$

$\leq 2 \mathrm{SM}$

$\leq 3 \mathrm{SM}$

Escolaridade

Nenhuma

16

50

11

Ensino Fundamental Completo

Ensino Médio Incompleto

Ensino Médio Completo

Ensino Superior Incompleto

Ensino Superior Completo

Tipos de Diabetes

Tipo 1

22

46

32

Frequência Relativa

(\%)

39

61

11

66

9

14

10

34

26

30

30

16

50

11

5

5

15

15

0

0

3

3

Tipo 2

22

Não sabe informar 
Com relação ao tipo de diabetes, $46 \%$ relataram apresentar o DM tipo 2, e entre este grupo, $41 \%$ ainda não sabem o que é a doença. Ao relacionar antecedentes familiares para a patologia, $70 \%$ dos entrevistados relataram a existência de um ou mais casos na família.

Com relação ao tipo de tratamento utilizado, nota-se que há frequência maior (53\%) do tratamento combinado de insulina e antidiabético oral (tabela 2). Em relação à prática de atividade física, $88 \%$ dos indivíduos entrevistados não realizam nenhum tipo de atividade.

Tabela 2. Tipo de tratamento utilizado pelos indivíduos com DM, usuários do CRA de Carapina - ES, 2010. $(n=100)$

Tipo de tratamento Frequência Absoluta (n) Frequência Relativa (\%) medicamentoso

\begin{tabular}{lcc}
\hline Insulina & 10 & 10 \\
Antidiabético oral & 35 & 35 \\
Insulina + antiabético oral & 53 & 53 \\
Nenhum & 2 & 2 \\
\hline
\end{tabular}

Dos 100 entrevistados, $98 \%$ acreditam na importância da alimentação adequada no tratamento do DM. No que diz respeito à importância do uso de adoçante para o tratamento, $52 \%$ relatam que este ajuda a conviver melhor com a doença e $31 \%$ considera ser indispensável.

Verifica-se ainda que $92 \%$ dos entrevistados receberam orientações sobre alimentação, e que nessa orientação, 97\% dos pacientes receberam também orientações para substituição do açúcar pelo adoçante.

Ao analisar qual profissional orientou os pacientes entrevistados quanto ao tratamento dietoterápico para o DM (figura 1), observa-se que o médico é o mais citado, seguido do nutricionista e do enfermeiro. E na opção “outros”, o único profissional citado foi o assistente social. 


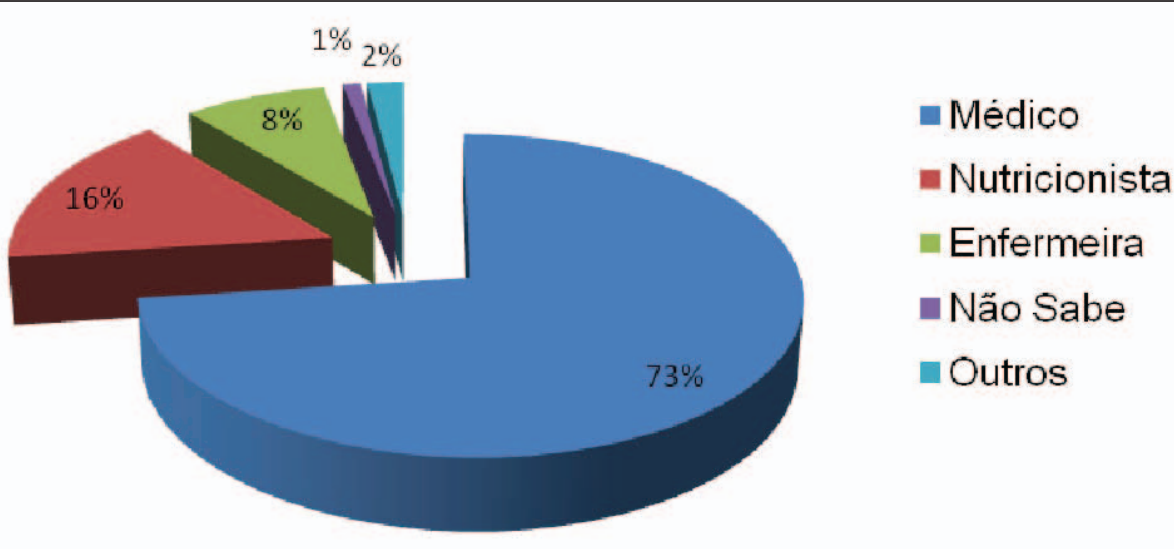

Figura 1. Distribuição percentual de profissionais que participaram da orientação alimentar dos indivíduos com DM, usuários do CRA de Carapina - ES, 2010. (n=92)

O hábito de não leitura de rótulos foi detectado em $63 \%$ dos indivíduos e os motivos para essa falta são diferentes, mas predominou a falta de interesse (40\%). No geral, a maioria dos indivíduos faz uso de adoçante (91\%), sendo que eles começaram a ingerir apenas após o diagnóstico do DM. A tabela 3 mostra que o motivo de utilizar este produto, para a maioria dos entrevistados, é a presença do DM $(51,65 \%)$, e o não-uso foi justificado principalmente por fazer mal $(55,56 \%)$ e não sentir necessidade.

Tabela 3. Justificativa para o uso e não-uso de adoçante por indivíduos com DM, usuários do CRA de Carapina - ES, 2010.

Frequência Absoluta (n) Frequência Relativa (\%)

Motivo do uso $(\mathrm{n}=91)$

Profissional recomendou

Por causa do DM

Porque gosta do sabor

Porque gosta de alimentos

doces

Outros

Motivo do não uso $(\mathrm{n}=9)$

Faz mal

Sabor ruim
5

43,96

47

51,65

1

1,1

1

1,1

2

2,19

55,56

4

44,44 
O tipo de adoçante mais consumido é na forma líquida, citado por $94 \%$ da amostra usuária de adoçante. Em relação à forma de uso, os entrevistados disseram fazer a contagem de gotas a serem consumidas $(78,02 \%)$, havendo ainda uma porcentagem considerável que não a faça (21,98\%). Dos 91 indivíduos entrevistados que fazem uso de adoçantes, $56 \%$ relataram ser preocupados com a quantidade ingerida e 57,14\% trocariam o adoçante por açúcar, caso pudessem.

Os indivíduos relataram maior consumo do adoçante ZeroCal® (65\%) e 80\% não sabem a composição do seu adoçante utilizado. No que se refere à seleção do adoçante, o sabor foi o principal critério utilizado (53\%), seguido de sugestão de amigos e parentes (11\%), preço (10\%) e marca (10\%). Pouco mais da metade da amostra já trocou de adoçante (63\%) e a justificativa principal foi o sabor desagradável do produto (46\%).

Entre os produtos mais consumidos, em que o açúcar é substituído por adoçante, está em primeiro lugar o refrigerante, citado por $53 \%$ entrevistados. O suco é o segundo mais consumido (47\%), e na opção “outros”, os mais citados foram: gelatina, iogurte, sorvete, barra de cereal e alguns doces, como cocada e goiabada. Em menor destaque, encontram-se geleia, pudim, chocolate e bolo.

Com relação à frequência de utilização, o refrigerante $(58,49 \%)$ e o suco $(53,19 \%)$ são consumidos semanalmente; já o pudim (71,43\%), o chocolate $(70 \%)$ e o bolo $(81,82 \%)$, mensalmente.

Quanto ao conhecimento acerca dos produtos diet e light, foi observado que apenas $41 \%$ dos indivíduos disseram saber a distinção entre eles. As respostas citadas pelos entrevistados para o produto diet foram: menos açúcar $(34,15 \%)$, isento de açúcar $(34,15 \%)$ e indicado para diabéticos $(31,7 \%)$; para o produto light, foram: menos gordura $(48,78 \%)$, indicado para emagrecimento $(19,51 \%)$, menos açúcar (12,2\%), não tem gordura (9,76\%) e indicado para diabéticos $(9,76 \%)$. Observa-se que existe uma confusão sobre o assunto, como mostram as respostas fornecidas pelos 41 indivíduos que disseram saber a diferença.

\section{Discussão}

A estratificação por sexo dos pacientes assistidos pelo CRA de Carapina que procuraram o Programa de Educação e Controle de Diabetes foi semelhante ao observado no estudo de Castro \& Franco, ${ }^{11}$ no qual predominou o sexo feminino, o que pode estar relacionado à maior preocupação e interesse desse grupo pelo atendimento e tratamento da patologia.

Também se constatou uma maior incidência de diabetes tipo 2, e destes há uma predominância da população idosa, o que pode ser justificado pelo estudo de Sousa, ${ }^{17}$ que afirma que esse tipo de diabetes está mais relacionado à população dessa faixa etária.

No que tange à escolaridade, mais da metade da população (66\%) em estudo não apresenta "nenhuma" ou "ensino fundamental incompleto", o que vem a dificultar o processo de educação 
nutricional aos indivíduos diabéticos, já que a compreensão se torna mais difícil. Este fato se assemelha ao observado no estudo de Sousa, ${ }^{17}$ no qual os indivíduos entrevistados apresentam renda familiar baixa, o que se relaciona com a escolaridade e a dificuldade de seguimento das orientações necessárias ao tratamento.

Em relação aos medicamentos, houve frequência maior do uso combinado de insulina com antidiabético oral (53\%), o que pode ser atribuído, de acordo com Oliveira, ${ }^{16}$ à dificuldade no controle da doença e estágio avançado da mesma. Foi relatado que o uso dos adoçantes só aconteceu após diagnóstico de DM, e que este é feito por obrigação, fato comprovado quando 52 indivíduos relataram que voltariam a consumir o açúcar se pudessem.

Aimportância do adoçante no tratamento da patologia foi descrita, relatando ser "indispensável" e "ajudar a conviver melhor com a doença", porém nem sempre conseguiam fazer uso do mesmo. Contudo, estes produtos não são imprescindíveis na vida desses indivíduos, mas ajudam a saciar o desejo pelo sabor doce e no controle glicêmico. ${ }^{18}$

Cada adoçante apresenta sua peculiaridade, como a limitação do uso do aspartame em altas temperaturas e a proibição de indivíduos hipertensos no uso do ciclamato de sódio, visto que ele apresenta sódio, por isso, a importância da identificação da composição do produto. ${ }^{16,19}$ Neste estudo, a identificação do edulcorante mais consumido pela população estudada se fez de acordo com o nome comercial do adoçante, visto que a grande parte não tinha conhecimento da composição do mesmo. As marcas mais citadas no estudo se relacionam com a reportagem de uma revista de circulação nacional, segundo Weinberg, ${ }^{20}$ que apresenta as marcas mais vendidas no mercado brasileiro, o que comprova que os adoçantes são mais conhecidos pelo nome comercial do que por sua composição.

Dessa forma, foi relatado maior consumo do adoçante ZeroCal®, e encontrou-se que aspartame, ciclamato e sacarina são os edulcorantes mais utilizados, não sendo possível saber a quantidade exata, visto que não foi informada a forma de apresentação desse adoçante.

Segundo Castro \& Franco, ${ }^{11}$ a combinação do ciclamato com a sacarina se dá no intuito de reduzir o sabor amargo da sacarina. Duarte \& Oliveira ${ }^{19}$ destacaram a sucralose e o esteviosídeo como os produtos mais indicados, por possuírem poder edulcorante considerável e menor sabor amargo, mas são os que apresentam preço mais elevado.

O trabalho desenvolvido por Castro \& Franco $^{11}$ também identificou que a maioria $(79,2 \%)$ dos diabéticos entrevistados prefere utilizar o adoçante na forma líquida de administração, sendo a mais adequada. E nos estudos de Castro \& Franco $^{11}$ e Sousa, ${ }^{17}$ respectivamente, 61,3\% e 87,8\% dos diabéticos entrevistados fazem a contagem das gotas do adoçante no momento do uso. De acordo com Duarte \& Oliveira, ${ }^{19}$ pouco mais da metade se preocupa com a quantidade de adoçante ingerida, fazendo esse controle mesmo não conhecendo a quantidade recomendada. 
No que se refere ao motivo da escolha dos adoçantes, houve concordância com os achados no estudo de Castro \& Franco, ${ }^{11}$ no qual o sabor chega como prioridade de escolha, seguido de sugestão de amigos e parentes, preço e marca, o que mostra que os indivíduos buscam alimentos de sua preferência. A influência da mídia, com propagandas, não teve tanta relação nesse grupo, e segundo Oliveira ${ }^{16} \mathrm{e}$ Castro \& Franco, ${ }^{11}$ tal influência pode ser associada a indivíduos mais jovens, que buscam melhora da qualidade de vida, vida saudável e controle da obesidade. Contrário ao dado encontrado no estudo de Oliveira, ${ }^{16}$ mais da metade dos diabéticos já trocaram de adoçantes, sendo essa atitude importante tanto na mudança da marca comercial, como do edulcorante utilizado.

Observou-se que existe equívoco quanto ao real significado dos termos diet e light, já que a maioria dos $41 \%$ que responderam ao questionamento não os conceituou da forma apropriada, resultado semelhante ao encontrado por Duarte \& Oliveira, ${ }^{19} \mathrm{o}$ que contribui para agravar o quadro da doença, já que existe consumo inadequado. O baixo perfil escolar da população entrevistada pode ter relação com essa realidade, já que a falta e/ou redução de calorias nos produtos podem ser desprezadas pela população de baixo nível de educação. ${ }^{16}$ Contudo, percebe-se a necessidade de melhoria nas informações sobre o uso e a composição desses produtos, visto que há um aumento da oferta e aquisição dos mesmos pelos indivíduos diabéticos.

O resultado encontrado na falta de leitura dos rótulos dos alimentos foi semelhante ao achado por Duarte \& Oliveira, ${ }^{19}$ segundo o qual vários fatores se relacionam com a falta de interesse pelos rótulos, como letras muito pequenas, segundo relatado por muitos indivíduos entrevistados, bem como falta de conhecimento e não entendimento das informações ali contidas. Tal achado era esperado, visto que esta é uma população com renda familiar baixa e pouca escolaridade, o que dificulta a compreensão e o processo de educação em diabetes, com mudança do estilo de vida. ${ }^{17}$ Assim sendo, o objetivo da rotulagem, de ajudar na escolha e no consumo dos produtos alimentícios, não é alcançada.

O consumo de produtos no qual o açúcar é substituído pelo adoçante não é feito por muitos dos entrevistados, pois não acreditam na troca e a ingestão faz com que eles se sintam mal. No entanto, informaram que não deixavam de consumir os alimentos ricos em carboidratos simples por serem diabéticos, o que contribui para as crises e aumento da glicemia, agravando o quadro de DM. ${ }^{17}$ No que tange ao consumo de adoçantes em locais fora de casa, pouco mais da metade informou que faz uso, pois muitos lugares ainda não apresentam o adoçante como uma opção, o que mostra falta de interesse, informação e conhecimento, por grande parte da população, das necessidades dos indivíduos diabéticos, já que esses produtos não são utilizados apenas por indivíduos que buscam perder peso.

A importância da alimentação no tratamento do DM foi reconhecida por quase todos os entrevistados (98\%), mas isso não resulta necessariamente no seguimento das orientações recebidas, 
já que muitos entrevistados não conseguem adequar à alimentação, o que dificulta o controle metabólico e, consequentemente, o tratamento, visto que o DM é uma patologia assintomática, o que faz com que o paciente acredite estar bem, pois não apresenta sintomas. Além disso, os profissionais de saúde devem atentar que a alimentação não é somente um fenômeno biológico, mas é influenciado pelos aspectos sociais, culturais e emocionais. ${ }^{16}$

O estudo de Péres e colaborados ${ }^{4}$ relata que diversos fatores podem influenciar esta questão, como os psicológicos, nível socioeconômico, escolaridade e sintomas de doenças. O nutricionista, mesmo com crescimento da profissão, não exerceu influência significativa na escolha do adoçante e nas orientações necessárias ao cuidado com a alimentação, o que pode ser justificado pela exclusão do profissional na equipe de atendimento aos diabéticos do CRA.

Em relação à prática de atividade física, os entrevistados relatam não terem o hábito de prática regular de exercício leve, moderado ou intenso, e estudos vêm demonstrando que a atividade física, juntamente com a mudança no estilo de vida e promoção de hábitos alimentares saudáveis, é eficaz para a prevenção e controle do diabetes, em especial o tipo 2, agindo no controle glicêmico. ${ }^{21}$

Recomenda-se comumente, para os diabéticos tipo 2, a realização de atividade aeróbica;; ${ }^{21}$ no entanto, o exercício resistido também é benéfico, em especial aos idosos, visto que, segundo Ciolac \& Guimarães, ${ }^{22}$ com a diminuição da força e massa muscular, há uma modificação prejudicial do metabolismo energético, e com a prática do exercício resistido há aumento da força e massa muscular, o que contribui para o controle glicêmico, com redução dos níveis de glicose e aumento do glicogênio muscular. Ressalta-se, entretanto, a importância de uma avaliação médica prévia, visto que o tipo de modalidade esportiva e a intensidade do exercício podem trazer prejuízo ao diabético.

A presença de amigos e familiares na vida dos indivíduos diabéticos contribui para o controle no tratamento da dieta, seguindo as orientações e prática da atividade física, o que auxilia na adesão aos cuidados necessários para o tratamento e controle dos sintomas e agravos, já que o desejo de se alimentar e a obrigação de controlar-se estão presentes a todo o momento na vida desse grupo.,19

\section{Conclusão}

Constatou-se que mesmo inseridos no programa de Diabetes oferecido pelo CRA de Carapina, com consultas com equipe multiprofissional e oficinas de orientações, os entrevistados apresentam pouco conhecimento sobre o DM, bem como, sobre a forma adequada do consumo de adoçante e sobre os tipos de edulcorantes. Dessa forma, torna-se necessário inserir informações a respeito do uso adequado e a composição dos mesmos, visto que o perfil educacional da população também é deficiente. 
A dificuldade de leitura dos rótulos implica a formação de consumidores mais conscientes e informados; assim, é necessário incentivar sua leitura, bem como modificar os rótulos com letras mais legíveis e linguagem simplificada, evitando informações errôneas que levam ao consumo inadequado dos produtos.

A inclusão do nutricionista na equipe multiprofissional, com a finalidade de orientar a respeito da importância das ações nutricionais e alimentares, ao tratamento e prevenção de agravos do DM, revela-se importante e necessária, além de contribuir na formação de maior conscientização dos indivíduos e promoção de hábitos alimentares saudáveis, na luta por melhora da saúde e qualidade de vida.

\section{Referências}

1. Santos JR, Enumo SRF. Adolescentes com diabetes mellitus tipo 1: seu cotidiano e enfrentamento da doença. Psicol reflex crit. 2003; 16(2): 411-25.

2. Canali ES, Kruel LFM. Respostas hormonais ao exercício. Rev paul educ fís. 2001 jul./dez.; 15(2): 141-53.

3. Sociedade Brasileira de Diabetes. Atualização brasileira sobre diabetes. Rio de Janeiro: 2010.

4. Péres DS, Franco LJ, Santos MA. Comportamento alimentar em mulheres portadoras de diabetes tipo 2. Rev Saúde Pública. 2006 abr.; 40(2).

5. Coeli CM, Ferreira LGFD, Drbal MM, Veras RP, Júnior KRC, Cascão AM. Mortalidade em idosos por diabetes mellitus como causa básica e associada. Rev Saúde Pública. 2002 abr.; 36(2): 135-40.

6. Alvarenga KF, Duarte JL, Silva DPC, Agostinho-Pesse RS, Negrato CA, Costa OA. Potencial cognitivo P300 em indivíduos com diabetes mellitus. Rev bras otorrinolaringol. 2005 mar./abr.; 71(2): 202-7.

7. Espírito Santo (Estado). Secretaria da Saúde. Diretrizes para manuseio da hipertensão arterial sistêmica e diabetes mellitus na rede de saúde pública. Vitória: 2008.

8. Accioly E, Saunders C, Lacerda EMA. Nutrição em Obstetrícia e Pediatria. 2. ed. Rio de Janeiro: Cultura Médica; 2009.

9. Mahan LK, Escott-Stump S. Krause Alimentos, Nutrição \& Dietoterapia. 10. ed. São Paulo: Roca; 2002.

10. Lottenberg AMP. Características da dieta nas diferentes fases da evolução do diabetes melito tipo 1. Arq bras endocrinol metab. 2008 mar.; 52(2).

11. Castro AGP, Franco LJ. Caracterização do consumo de adoçantes alternativos e produtos dietéticos por indivíduos diabéticos. Arq bras endocrinol metab. 2002 jun; 46(3): 280-7. 
12. Agência Nacional de Vigilância Sanitária (Brasil). Portaria n. 29, de 13 de janeiro de 1998. Aprova o Regulamento Técnico referente a Alimentos para Fins Especiais. Diário Oficial da União 30 de março de 1998. Disponível em: http://portal.anvisa.gov.br/wps/wcm/connect/7f683d00474575d2 83bed73fbc4c6735/PORTARIA_29_1998.pdf?MOD=AJPERES

13. Agência Nacional de Vigilância Sanitária (Brasil). Portaria n. 38, de 13 de janeiro de 1998. Aprova o Regulamento Técnico referente a Adoçantes de Mesa, constante do anexo desta Portaria. Diário Oficial da União 15 de janeiro de 1998. Disponível em: http://portal.anvisa.gov.br/wps/wcm/conn ect/3bfc2e80474579448655d63fbc4c6735/PORTARIA_38_1998.pdf?MOD=AJPERES

14. Torloni MR, Nakamura MU, Megale A, Sanchez VHS, Mano C, Fusaro AS, et al. O uso de adoçantes na gravidez: uma análise dos produtos disponíveis no Brasil. Rev bras ginecol obstet. 2007; 29(5): 267-75.

15. Paiva ES. Adoçantes artificiais: novos limites, novas substâncias e benefícios ao nosso alcance. Food Ingredients Brasil. 2008; (5).

16. Oliveira PB. Consumo de adoçantes e produtos dietéticos por indivíduos com diabetes mellitus tipo 2, atendidos pelo Sistema Único de Saúde em Ribeirão Preto - SP. [Dissertação]. Universidade de São Paulo. Departamento de Medicina Social. Faculdade de Medicina: Ribeirão Preto; 2009.

17. Sousa G. Uso de adoçantes dietéticos por pessoas diabéticas. [Dissertação]. Enfermagem Fundamental. Escola de Enfermagem de Ribeirão Preto: São Paulo; 2006.

18. Goveia GR, Bruno LPC, Pascali PM. Contagem de carboidratos \& monitorização: 101 respostas. São Paulo: Preventa; 2003.

19. Duarte MJD, Oliveira TC. Conhecimento e forma de consumo de adoçantes e produtos dietéticos em indivíduos hipertensos e diabéticos do programa hiperdia de um PSF do município de Itabira, MG. Revista Digital de Nutrição. 2008 fev./jul.; 2(2).

20. Weinberg M. Os testes dos adoçantes. Revista Veja 2006 jan.; (3):98-9.

21. Ciolac EG, Guimarães GV. Artigo de revisão exercício físico e síndrome metabólica. Rev bras med esporte. 2004 jul./ago.; 10(4).

22. Ciolac EG, Guimarães GV. Importância do exercício resistido para o idoso. Rev Soc Cardiol Estado de São Paulo. 2002; 12: 15-26. 
